REVISTA DE DERECHo UNED, NÚM. 7, 2010

\title{
EL DERECHO CONSTITUCIONAL A UN MEDIO AMBIENTE SALUDABLE EN RUMANIA
}

\author{
Livia Mocanu \\ Olivian Mastacan
}

Profesores Titulares de Derecho, Universidad «Valahia» de Targoviste Facultad de Ciencias Jurídicas, Sociales y Políticas

Resumen. El derecho del medioambiente saludable se inscribe entre los más recientemente reconocidos y garantiza diversos derechos fundamentales del hombre. Las constituciones que prevén un derecho fundamental del medioambiente contienen disposiciones precisas y claras, como elementos que marcan sus significados.

En Rumania, el derecho constitutional a un medioambiente saludable permitió una mejor armonización y una correlación con las reglamentaciones europeas e internacionales, lo que conlleva una exigencia legislativa de la estrategia de protección medioambiental y también de tratado de adhesión a la Unión Europea.

\begin{abstract}
The right of the healthy environment registers between more recently recognized and guarantees diverse fundamental rights of the man. The constitutions that foresee a fundamental right of the environment contain precise and clear dispositions, as elements that mark his meanings.

In Romania, the right constitutional a healthy environment allowed a better harmonization and a correlation with the European and international regulations, which carries a legislative exigency of the strategy of environmental protection and also of agreement of adhesion to the European Union.
\end{abstract}

Palabras Clave : Derecho, medioambiente, constitución.

Key Words: Law, environment, constitution. 


\section{INTRODUCCIÓN}

El reconocimiento y la garantía del derecho de cada persona a un medio ambiente saludable, como derecho fundamental, ha constituido uno de los problemas jurídicos de una especial significación en el contexto de la armonización de la legislación interna con el derecho comunitario del medio ambiente.

El proceso de asimilación del acquis comunitario del medio ambiente comenzado en el contexto de la preparación de la adhesión de Rumania a la Unión Europea ha contribuido esencialmente a la consolidación de las reglamentaciones nacionales en el dominio del medio ambiente.

La evolución de las reglamentaciones nacionales en este dominio y las consecuencias de la incluir el derecho a un medio ambiente saludable en la Constitución hace el objeto de nuestro análisis.

\section{EVOLUCIÓN LEGISLATIVA.}

El proceso de consagrar y de garantizar el derecho fundamental a une medio ambiente saludable ha registrado en nuestro país una evolución cuyos momentos han sido influidos significadamente por la ratificación de los documentos internacionales en el dominio y por la preparación de la adhesión a la Unión Europea ${ }^{1}$.

Aunque las primeras menciones constitucionales de la problemática ecológica han ocurrido en los estados ex-comunistas ${ }^{2}$, la Constitución de Rumania de 21 de Agosto de 1965 no contenía ninguna disposición en materia.

La primera Conferencia mundial de ONU (Junio de 1972, Stockholm) ha estimulado las preocupaciones de los estados en la dirección de consagración legislativo -constitucional de los problemas concertando la protección del medio- ambiente. ${ }^{3}$

\footnotetext{
${ }^{1}$ Ampliamente, verse, Mircea Dutu, El reconocimiento y garantía del derecho fundamental al medio ambiente en Rumania, en la Revista Dreptul no. 6/2004, p. 99101; Mircea Dutu, Tratado del derecho del medio ambiente, Edición $3^{\mathrm{a}}$, Casa Editorial C.H. Beck, Bucarest, 2007, p. 306-309.

${ }^{2}$ R.M. Mateo, Tratado de derecho ambientat, tomo IV (Actualización), Edisofer, s. 1., Madrid, 2003, p. 45.

${ }^{3}$ Declaración de Stockolm de 1972 ha consagrado, por la primera vez, el derecho humano a un medio ambiente "cuya cualidad le permita vivir con dignidad y bienestar». La idea es retomada y afirmada con más precisión en la Carta Africana sobre los
} 
En este contexto, ha sido adoptada en Rumania primera ley - marco con respecto a la protección del medio ambiente, la Ley no. 9/19734. Con todas sus virtudes por aquel momento tampoco este acto normativo no ha reglamentado el derecho a un medio ambiente saludable.

Después de los años 1990 el reconocimiento y la garantía del derecho a un medio ambiente saludable se convirtió en un problema de actualidad, constituyéndose en un debate intensa en la doctrina jurídica $^{5}$. Con todo esto la Constitución de Rumania de 1991 no lo ha consagrado como derecho fundamental.

Es cierto que la ley fundamental comprendía una serie de previsiones significantes en el dominio, instituyendo la obligación del estado de asegurar, entre otras «el rehacimiento y la protección del medio ambiente, como el mantenimiento del equilibrio ecológico» conforme a las disposiciones del art. 134 apartado (2), letra e, pero un tal reglamentación parcial no equivalía con un reconocimiento expreso del derecho fundamental a un medio ambiente equilibrado ecológico ${ }^{6}$.

La falta de la previsión constitucional en materia ha sido completada por Ley no. 137/1995 con respecto a la protección del medio ambiente ${ }^{7}$, acto normativo que ha consagrado una concep-

derechos humanos y de los pueblos adonde se especifica que: «todos los pueblos tienen el derecho a un medio ambiente general satisfactorio, favorable a su desarrollo» (art. 24). Ampliamente, sobre las conferencias mundiales en el dominio de la protección del medio ambiente y de los principales documentos adoptados, verse Daniela Marinescu, Tratado del derecho del medio ambiente, Edición $\mathrm{II}^{\mathrm{a}}$, revisada y añadida, Casa Editorial Universul Juridic, Bucarest, 2007, p. 19-22.

${ }^{4}$ Publicada en el Boletín Oficial no. 91 de 23 de junio de 1973.

${ }^{5}$ Mircea Dutu, Sobre la necesidad del reconocimiento y las significaciones del derecho fundamental del hombre a un medio ambiente saludable, en la Revista Dreptul no. 912/1990, p. 41-49; Ernest Lupan, El derecho a un medio ambiente no contaminado, «Studia Universitatis Babes - Bolyai» Iurisprudentia, 1, 1993, p. 49 y siguientes; Ernest Lupan, de nuevo sobre el derecho a un medio ambiente saludable», «Studia Universitatis Babes - Bolyai» Iurisprudentia, 1-2, 1995; M. Ghezali, Les nouveaux droits fondamentaux de l'homme, en el tomo Vers un nouveau droit de l'environnement?, Reunión International de Droit Comparé de L'environnement, Limoges, 2003, p. 93-98.

${ }^{6}$ En el sentido que por estas disposiciones la Constitución de 1990 consagraba «indirectamente, por vía de consecuencia» un derecho fundamental de los ciudadanos aferente, verse Mircea Dutu, El derecho del medio ambiente, Casa Editorial Gamian, Bucarest, 1993, p. 62. En el sentido contrario, verse Ernest Lupan, El derecho del medio ambiente, Casa Editorial Lumina Lex, Bucarest 2001, p. 29.

${ }^{7}$ Publicada en el Boletín Oficial no. 304 de 30 de diciembre de 1995, republicada en el Boletín Oficial no. 70 de 17 de diciembre de 2002, modificada y completada por O.U.G. no. 91 de 20 de junio de 2002, publicada en el Bo,etín Oficial no. 465 de 28 de junio de 2002, aprobada con modificaciones y suplementos por la Ley no. 294 de 27 de junio de 2003, publicada en el Boletín Oficial no. 505 de 14 de julio 2003. Ley no. 137/1995 ha sido abrogada por O.U.G. no. 195/2005. 
ción moderna en materia y ha definido la protección del medio ambiente como «objetivo de interés público mayor» (art. 1).

En el art. 5 de la Ley no. 137/1995, el legislador ha consagrado expresamente, como derecho subjetivo, el derecho a un medio ambiente saludable y ha previsto las garantías legales para su ejercitación. Bajo la fórmula: «El estado reconoce a todas las personas el derecho a un medio ambiente saludable...», la ley marco en materia ha representado un instrumento jurídico especial para la consagración legislativa y para la garantía de este derecho.

En el contexto de la finalización del proceso de armonización de la legislación rumana con el acquis comunitario en el dominio de la protección del medio ambiente, se elabora una nueva reglamentación marco en materia.

Así, instituyendo los principios y los elementos estratégicos del derecho comunitario en el dominio del derecho del medio ambiente, el Orden de Urgencia del Gobierno (O.U.G.) no. 195/2005 reglamenta el derecho a un medio ambiente saludable en las disposiciones del art. 5, conforme con los cuales: «El estado reconoce a cualquier persona el derecho a un medio ambiente saludable y equilibrado ecológico...».

De aquí resulta que el derecho a un medio ambiente saludable es no solamente reconocido por la ley - marco pero también protegido y garantizado efectivamente, el mismo artículo 5 de O.U.G. no. 195/2005 estableciendo también las garantías legales para su ejercitación. En este contexto, la ley garantiza:

- el acceso a la información concertando el medio ambiente, con respecto a las condiciones de confidencialidad previstas por la legislación vigente,

- el derecho de asociación en organizaciones para la protección del medio ambiente;

- el derecho de ser consultado en el proceso de tomar decisiones concertando el desarrollo de la política y de la legislación del medio ambiente, la expedición de los actos de reglamentación en el dominio, la elaboración de los planes y de los programas;

- derecho de dirigirse, directamente o por medio de las organiza-

${ }^{8}$ Publicada en el Boletín Oficial no. 1.196 de 30 de diciembre 2005, O.U.G. no. 195/2005 ha sido aprobada con modificaciones y suplementos por la Ley no. 265/2006 publicada en el Boletín Oficial no. 586 de 6 de julio de 2006. Se le han llevado modificaciones y suplementos por O.U.G. no. 57 de 20 de junio de 2007 y por O.U.G. no. 114 de 17 de octubre de 2007. 
ciones para la protección del medio ambiente, a las autoridades administrativas y/ o judiciales, después el caso independientemente si se ha producido o no un perjuicio;

- derecho a indemnización para el perjuicio sufrido.

También en la categoría de las reglamentaciones sectoriales con respecto a la protección del medio ambiente está enunciado el derecho a un medio ambiente saludable. Está el caso del Orden de Urgencia del Gobierno no. 243/2000 que reglamenta la protección de la atmósfera ${ }^{9}$ y que instituya como objeto prioritario de la estrategia nacional en el dominio, asegurar «el derecho de cada persona a un medio ambiente de cualidad» [art. 1 apartado (2)].

También, como una particularización del derecho constitucional a la información ${ }^{10}$ y como resultado de la transposición de las directivas de la Unión Europea, por medio de la Decisión del Gobierno no. 878/2005 de acceso del público a la información sobre el medio ambiente ${ }^{11}$ se ha reglamentado el acceso de cualquier persona a la información concertando el medio ambiente detenida por o para las autoridades públicas, instituyendo un régimen jurídico correspondiente para ejercitar este derecho.

\section{CONTRIBUCIÓN DEL DERECHO COMUNITARIO.}

$\mathrm{Al}$ nivel europeo, después la elaboración de la Convención europea de los derechos humanos (Roma, 4 de Noviembre de 1950) cual no comprende referencias a los problemas de medio puesto que en este tiempo «la crisis ecológica» no había manifestado, la jurisprudencia de la Comisión y de la Corte Europea de los Derechos Humanos ha establecido que la deterioración del medio puede afectar los derechos reconocidos por la Convención, incluso el derecho a vida privada y a la vida de familia, como el derecho a la propiedad ${ }^{12}$.

${ }^{9}$ Publicada en el Boletín Oficial no. 633 de 6 de diciembre de 2000, O.U.G. no. 243/2000 ha sido aprobada por la Ley no. 655/2001.

${ }^{10}$ Art. 31 de la Constitución de Rumania.

${ }^{11}$ Publicada en el Boletín Oficial no. 760 de 22 de agosto de 2005.

12 En este caso, CEDO ha recurrido a la técnica pretoriana de la "protección por medio del rebote», cual permitió la extensión de la protección de ciertos derechos garantizados por la Convención de derechos que no son expresamente previstos por estos. Para desarrollos, verse Mircea Dutu, El reconocimiento y garantía del derecho fundamental al medio ambiente en Rumania, en la Revista Dreptul no. 6/2004, p. 101106; Mircea Dutu, operas citadas Tratado..., p. 287-294; F. Sudré, La protection du droit à l'environnement par CEDH, en el tomo La Communauté européene et l'environnement, La documentation Française, 1997, p. 209 y siguientes. 
En el derecho comunitario, el Tratado de Maastricht celebrado en el año 1993 ha incorporado y establecido, aunque por vía de referencia indirecta, el derecho fundamental a medio ambiente entre los derechos humanos reconocidos y garantizados al nivel de la orden jurisdiccional comunitaria ${ }^{13}$.

Relevantes bajo el aspecto de nuestro análisis son las reglamentaciones comunitarias concertando las garantías procedimentales del derecho a un medio ambiente saludable en las condiciones en que, al nivel del derecho comunitario, no se reconoce expresamente, independiente, el derecho fundamental a un medio ambiente saludable de los ciudadanos de la Unión Europea. Tenemos en cuenta las disposiciones del derecho comunitario derivado cual, bajo varias formas, reglamenta el acceso a la información, la participación del público de tomar decisiones y el acceso en la justicia. Ellas están el resultado de la firma, por la Comunidad Europea, de la Convención de Aarhus con respecto al acceso a la información, la participación del público a tomar la decisión y el acceso en la justicia en los problemas del medio ambiente, adoptada en el día de 25 de Junio de 1998 por la IV $^{\text {a }}$ Conferencia de los ministros un «Medio ambiente para Europa ${ }^{14}$. Retenemos aquí la Directiva no. 2003/4/CE del Parlamento Europeo y del Consejo concertando el acceso del público a la información del medio ambiente ${ }^{15}$ y el Reglamento (CE) no. 1367/2006 del Parlamento Europeo y del Consejo, de 6 de Septiembre de 2006, concertando la aplicación por las instituciones y los órganos de la Comunidad Europea de las disposiciones de la Convención de Aarhus. Los documentos tienen en vista el cumplimiento de las obligaciones que resultan de la Convención de Aarhus por los organismos comunitarios, estableciendo un juego de medidas adecuadas.

El derecho comunitario comprende también normas referentes al contenido material del derecho a un medio ambiente saludable por las directivas referentes a la cualidad del medio ambiente ${ }^{16}$ y las que visan el agua ${ }^{17}$.

${ }^{13}$ Ampliamente, verse, Mircea Dutu, El derecho comunitario del medio ambiente, Casa Editorial Económica, Bucarest, 1997, p. 67 y siguientes.

${ }^{14}$ Convención de Aarhus ha sido ratificada por Rumania por la Ley no. 86/2000 publicada en el Boletín Oficial no. 224 de 22 de Mayo de 2000.

${ }^{15}$ Directiva abroga Directiva 90/313/CEE (JO L 41/26 de 14 de Febrero de 2003).

${ }_{16}$ Directiva no. 1999/30/CE del Consejo de 22 de Abril de 1999, relativa a la fijación de los valores límites para anhídrido sulfuroso, dióxido de nitrógeno y óxidos de nitrógeno, partículas de plomo, en el aire ambiente (JOCE L 163 de 29 de Junio de 1999).

${ }^{17}$ Directiva 98/83/CE del Consejo de 3 de Noviembre de 1998, relativa a la cualidad de los aguas destinados al consumo humano (JOCE L 330 de 5 de Diciembre de 1998). 
El derecho a un medio ambiente saludable es un principio general del derecho comunitario cual, en su aplicación, tiene en cuenta la constitución de este derecho en los estados miembros de la Unión Europea $^{18}$.

\section{EL RECONOCIMIENTO Y LA GARANTÍA DEL DERECHO FUNDAMENTAL A UN MEDIO AMBIENTE SALUDABLE EN LA CONSTITUCIÓN REVISADA.}

El acceso de Rumania a las instituciones internacionales con preocupaciones mayores en el dominio del medio ambiente ${ }^{19}$ el desencadenamiento y la finalización del proceso de adhesión a la Unión Europea y la aproximación de la legislación interna con el derecho comunitario del medio ambiente ${ }^{20}$ han estimulado el proceso de constitución del derecho humano a un medio ambiente saludable.

Por el referéndum nacional de 18-19 de Noviembre de 2003 ha sido aprobada la Ley no. 429/2003 de revisión de la Constitución ${ }^{21}$. En la elaboración de este acto normativo es importante para mencionar el papel que tuvo la Decisión no. 148/200322 de la Corte Constitucional concertando la constitución de la propuesta legislativa de revisión de la ley fundamental con lo cual: Para asegurar la realización del propósito de la propuesta legislativa, la Corte considera que es necesario insertaren el Capítulo II del Título II de la Constitución, el derecho humano a un medio ambiente saludable...».

En consecuencia, ha sido introducido, en el Título II - «Los derechos, las libertades y las obligaciones fundamentales», Capítulo II - «Los derechos y las libertades fundamentales», un nuevo artículo (35), intitulado «El derecho a un medio ambiente saludable». Conteniendo una reglamentación estructurada en tres apartados: «(1) El estado reconoce el derecho de cualquier persona a un medio ambiente saludable y equilibrado ecológico». (2) El estado asegura el cuadro legislativo para la ejercitación de este derecho. (3) Las personas físicas y jurídicas tienen la obligación

1823 de estados miembros de la Unión Europea de los 27 reconocen y garantizan en la constitución el derecho fundamental del hombre a un medio ambiente saludable

${ }^{19}$ Consejo de Europa, 1991; Agencia Europea para el medio ambiente, 1998.

${ }^{20}$ Ampliamente, sobre el Derecho comunitario del medio ambiente, verse Mircea Dutu, operas citadas p. 162-180; Mircea Dutu, El derecho del medio ambiente, Tratado, tomo II, Casa Editorial Económica, Bucarest, p. 223-322; Dumitru Mazilu, Derecho comunitario del medio ambiente, Casa Editorial Lumina Lex, Bucarest 2006.

${ }^{21}$ Publicada en el Boletín Oficial no. 758 de 29 de Octubre de 2003.

22 Publicada en el Boletín Oficial no. 317 de 12 de Mayo de 2003. 
de proteger y mejorar el medio ambiente», el artículo 35 de la Constitución consagra y garantiza expresamente, independiente, el derecho fundamental del hombre a un medio ambiente saludable.

No podemos decir la misma cosa sobre la obligación colectiva que tiene cualquier persona física y jurídica de proteger y mejorar el medio ambiente, cual insertada también en el artículo 35, no tiene más una existencia independiente como las otras obligaciones fundamentales de los ciudadanos ${ }^{23}$. También, este hecho exprime el específico de la nueva reglamentación constitucional cual especifica junto al derecho del hombre a un medio ambiente saludable la obligación de todas las personas físicas y jurídicas de proteger y mejorar el medio ambiente, justamente como una garantía especial de este derecho. De acuerdo con la doctrina de especialidad ${ }^{24}$ retenemos que la afirmación expresa de la obligación de proteger el medio ambiente, levanta la protección del medio ambiente «al rango de obligación fundamental».

Además, empezando de la nueva reglamentación constitucional y teniendo en cuenta las exigencias del proceso de integración europea, O.U.G. no. 195/2005 regula en el art. 5 el derecho humano a un medio ambiente saludable y las garantías legales para su ejercitación.

En el mismo tiempo, de acuerdo al art. 6 de O.U.G. no. 195/2005, la protección del medio ambiente está considerada como una obligación y una responsabilidad de las autoridades de la administración pública central y local, como de todas las personas físicas y jurídicas.

Indiferentemente de la reglamentación comprendida en la Constitución o en la ley - marco de la protección del medio ambiente, el derecho fundamental a un medio ambiente saludable y equilibrado ecológico es reconocido a cualquier persona, lo que significa que el ser humano ${ }^{25}$, en su hipótesis de individuo, colectividad y «especie entre las especies», es el titular de este derecho.

${ }^{23}$ Colocadas separadamente, en la estructura del Título II, Capítulo III de la Constitución.

${ }^{24}$ Mircea Dutu, operas citadas Tratado..., p. 313

${ }^{25}$ En la literatura extranjera de especialidad se han formulado dos tesis referentes a la cuestión; quien es el titular del derecho a un medio ambiente saludable». Los adeptos de la tesis «finalidades humanas» han sostenido que este derecho no puede pertenecer sólo al individuo, el titular y el único beneficiario de este derecho siendo el hombre. Verse, en este sentido, J. de Falafosse, Le droit de l'environnement. Le droit à la nature, Paris, 1973, p. 250. Al contrario, otros autores, han sostenido que la naturaleza (que incluye el hombre) tiene el derecho de ser protegida. Verse, C.M. Vardot, Declaration des droits de la nature, Paris, 1973. Desde el punto de vista jurídico, es indiscutible que sólo el ser humano puede ser el titular del derecho a un medio ambiente saludable, y no la naturaleza o el medio ambiente. 
En consecuencia, la ejercitación de este derecho puede tener lugar, sea individualmente, sea de modo colectivo; en la última hipótesis, un papel importante lo tienen las organizaciones no gubernamentales ecológicas - las O.N.G., apolíticas, voluntarias, sin un propósito patrimonial. La participación pública es fuerte condicionada por el derecho de petición garantizado en el art. 51 de la Constitución, derecho de cual goza tanto los ciudadanos vistos como individuos, como las organizaciones legalmente constituidas.

Para establecer el contenido del derecho a un medio ambiente saludable, una significación especial lo representa la relación entre el derecho de propiedad y el derecho del medio ambiente. En este sentido, la Constitución revisada establece en el art. 44 apartado (7) que: «El derecho de propiedad obliga a respetar las tareas concertando la protección del medio ambiente y asegurar una buena vecindad, como respetar las otras obligaciones cual, de acuerdo a la ley o al costumbre pertenecen al propietario». En virtud de estas previsiones constitucionales, todas las reglamentaciones jurídicas en materia de la propiedad están bordeadas en sus efectos por las previsiones de la legislación concertando la protección del medio ambiente. Como justamente se afirmó en la literatura de especialidad ${ }^{26}$, nos encontramos en la presencia de una servidumbre constitucional de utilidad pública, con un régimen jurídico especial adaptado a la necesidad pública de protección del medio ambiente.

\section{CONCLUSIONES}

La constitución del derecho al medio ambiente es el fruto de la asimilación de un nuevo derecho fundamental, comprendido en la «tercera generación», la de «los derechos de solidaridad», caracterizado por una dinámica especial en lo que concierne su reconocimiento y su garantía jurídica. Así como se muestra en la doctrina, si el derecho del medio ambiente resulta del interés común de la humanidad, podemos considerar que este interés se refleja en los derechos reconocidos al individuo ${ }^{27}$. Como hemos visto, tanto las reglamentaciones internacionales en materia como las preocupaciones

\footnotetext{
${ }^{26}$ Mircea Dutu, operas citadas Tratado..., p. 321-322.

${ }^{27}$ Alexandre Kiss, Droit internationale de l'environnement, Paris, Pedone, 1989, p. 20 y siguientes; Mircea Dutu, El derecho internacional y comunitario del medio ambiente, Casa Editorial Económica, Bucarest, 1995, p. 66-71; Alexandre Kiss, D. Shelton, Traite de droit europeen de l'environnement, Casa Editorial Frison - Roche, Paris, 1995, p. 47-50.
} 
existentes al nivel del derecho comunitario han abierto la perspectiva de constituir en plano interno el derecho a un medio ambiente saludable y equilibrado ecológico. Los principios y las normas jurídicas cual accionan al nivel de la Unión Europea han influido positivamente las preocupaciones del legislador constitucional en el sentido del reconocimiento y de la garantía expresa del derecho al medio ambiente, como derecho fundamental.

Por lo demás, la integración de Rumania en la Unión Europea no ha sido sólo un desiderátum nacional, pero también un proceso complejo de transformación interna cual tuvo una significante componente legal-jurídica ${ }^{28}$.

El reconocimiento y la garantía constitucional del derecho a un medio ambiente saludable en nuestro país amplifica las obligaciones de las autoridades públicas para proteger el medio ambiente, ofrece a los instancias medios nuevos para la reparación de los perjuicios ecológicos y la sanción de los ofensas al medio ambiente, permite una mejor armonización entre los varios niveles de reconocimiento y garantía del derecho fundamental al medio ambiente, realizando una correlación necesaria entre la legislación nacional, por una parte y la reglamentación internacional (entendiendo aquí también la comunitaria), por otra.

${ }^{28}$ Lucian Miulescu, Las principales características de las relaciones entre el derecho comunitario europeo y los derechos nacionales de los estados miembros. Consecuencias para el derecho rumano, en la perspectiva de la adhesión de Rumania en la Unión Europea, en la revista Dreptul no. 12/2000, p. 27. 